\title{
Paraganglioma of the urinary bladder with chromosome duplications detected by fluorescence in situ hybridization in urine exfoliated cells: A case report
}

\author{
CHUNGUANG YANG, ZHENG LIU, RUZHU LAN, ZHIHUA WANG, ZHIQUAN HU, \\ ZHIQIANG CHEN and ZHANGQUN YE \\ Department of Urology, Tongji Hospital, Tongji Medical College, \\ Huazhong University of Science and Technology, Wuhan, Hubei 430030, P.R. China
}

Received December 11, 2014; Accepted October 21, 2015

DOI: $10.3892 / 01.2015 .3941$

\begin{abstract}
Paragangliomas of the urinary bladder are rare neoplasms derived from chromaffin tissue with a chromosomal imbalance. Their preoperative diagnosis and assessment of malignant potential remain significant challenges for urologists. The current report presents the case of a 34-year-old male who presented with a history of paroxysmal gross hematuria lasting for 7 months. Chromosome duplications were detected by fluorescence in situ hybridization (FISH) in urine exfoliated cells, and the diagnosis was confirmed by histopathology following a transurethral resection. To the best of our knowledge, this is the first reported case of urinary bladder paraganglioma in which chromosomal duplications were detected by FISH in urine exfoliated cells. This may be helpful to its differential diagnosis and malignant potential determination.
\end{abstract}

\section{Introduction}

Derived from chromaffin tissue of the sympathetic nervous system, paragangliomas have the potential to secrete catecholamines, accounting for several of the predominant symptoms of functional paragangliomas. The common presentation of urinary bladder paraganglioma is painless haematura, paroxysmal hypertension, headache, palpitation and anxiety (1). Metastasis to the lymph nodes and invasion of adjacent organs indicate the malignant potential (2). Urinary bladder paragangliomas are rare neoplasms constituting only $0.06 \%$ of all bladder tumors (1). The preoperative diagnosis and assessment

Correspondence to: Professor Ruzhu Lan, Department of Urology, Tongji Hospital, Tongji Medical College, Huazhong University of Science and Technology, 1095 Jiefang Avenue, Wuhan, Hubei 430030, P.R. China

E-mail: ruzhulan@yahoo.com.cn

Key words: urinary bladder, fluorescence in situ hybridization, chromosome duplication, paraganglioma of malignant potential of paragangliomas, particularly non-functional paragangliomas, present significant challenges for urologists (3). Tumor-specific patterns of chromosomal imbalances characterize almost all forms of neoplasm, and have been used in the diagnosis and malignancy determination of many tumors (4). For example, multiprobe fluorescence in situ hybridization (FISH) analysis of exfoliated cells in the urine has been approved for use in the detection of recurrent urothelial carcinoma and the evaluation of hematuria, as FISH exhibits a higher sensitivity compared with conventional cytology in detecting urothelial carcinoma (81 vs. 58\%) (5).

To the best of our knowledge, the current study is the first report of a case of a urinary bladder paraganglioma in which chromosome duplications were detected by fluorescence in situ hybridization (FISH) in exfoliated cells in the urine. This may be helpful to its preoperative diagnosis and malignant potential determination.

\section{Case report}

A 34-year-old male complaining of paroxysmal gross hematuria lasting for 7 months was admitted to Tongji Hospital (Wuhan, China). His medical and family history were unremarkable. General examination, including blood pressure and heart rate, yielded results within the normal range. Abdominal computed tomography (CT) imaging revealed a $3.6 \times 3.9-\mathrm{cm}$ mass on the dome of the bladder, which exhibited intense uniform enhancement on a contrast study (Fig. 1A). There was no indication of lymph node involvement or invasion to the surrounding structures. Cystoscopy revealed a $3 \times 4-\mathrm{cm}$ submucosal neoplasm on the dome of the bladder, partly covered by normal urothelium (Fig. 1B). A biopsy of the neoplasm was conducted, revealing a highly vascular tumor. FISH of exfoliated cells in the urine indicated the presence of duplications of chromosomes 3 and 17 (Fig. 1C and D).

Upon diagnosis of the bladder tumor, a transurethral resection was performed. During the surgery, no hypertension occurred. On microscopic examination of the resected specimen, nested spindle cells were observed (Fig. 2A). Immunohistochemical staining revealed positive reactivity for chromogranin A, S-100 protein, CD56 and synaptophysin, 
A

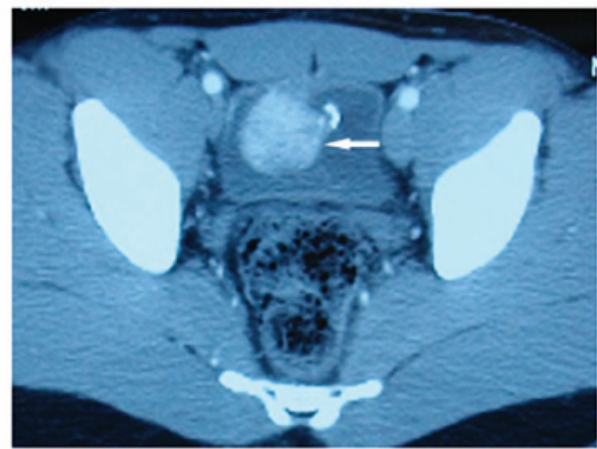

C

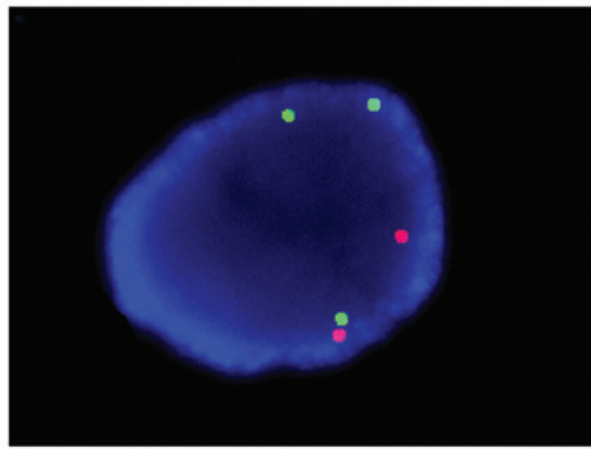

B

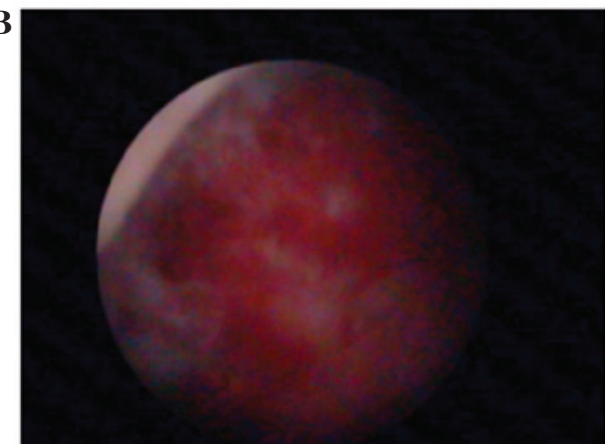

D

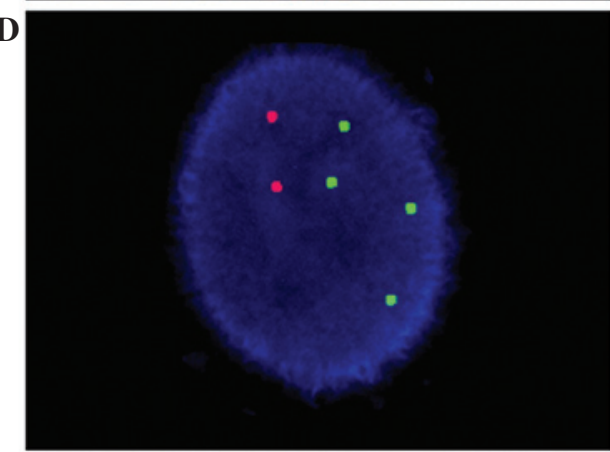

Figure 1. (A) Computed tomography of a 34-year-old male patient revealed a 3.6x3.9-cm mass on the dome of the bladder with intense uniform enhancement (arrow). (B) Cystoscopy revealed a 3x4-cm submucosal neoplasm on the dome of the bladder with ulceration and a rich blood supply. (C) Fluorescence in situ hybridization of chromosomes 17 (green) and 9p16 (red) in urine exfoliated cells revealed polysomy of chromosome 17. (D) Fluorescence in situ hybridization of chromosomes 3 (green) and 7 (red) in urine exfoliated cells revealed polysomy of chromosome 3.

A

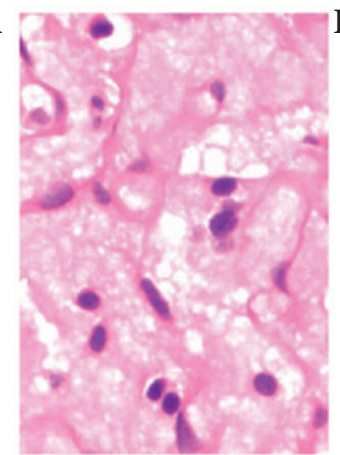

B

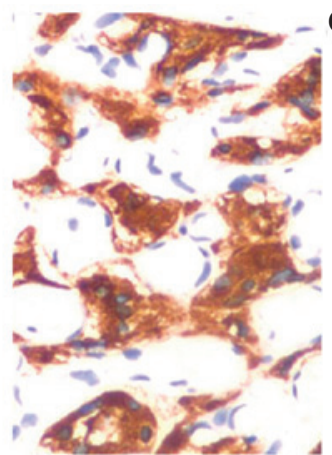

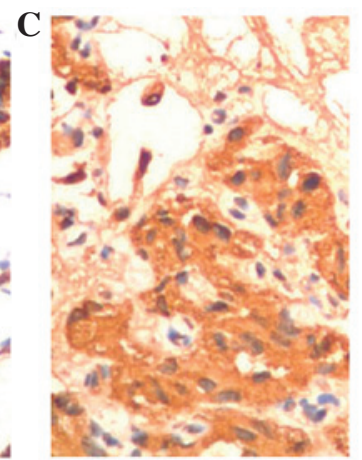

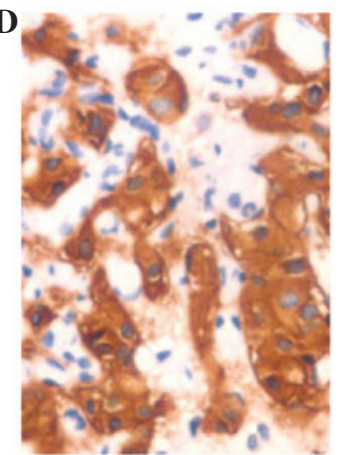

Figure 2. (A) Microscopic examination revealed nested spindle cells (hematoxylin and eosin staining; magnification, $\mathrm{x} 400$ ). The tumor cells were positive for (B) synaptophysin, (C) chromogranin A and (D) CD56 (magnification, x200).

A

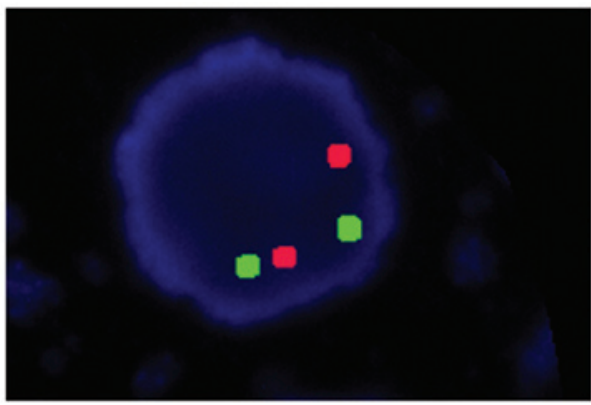

B

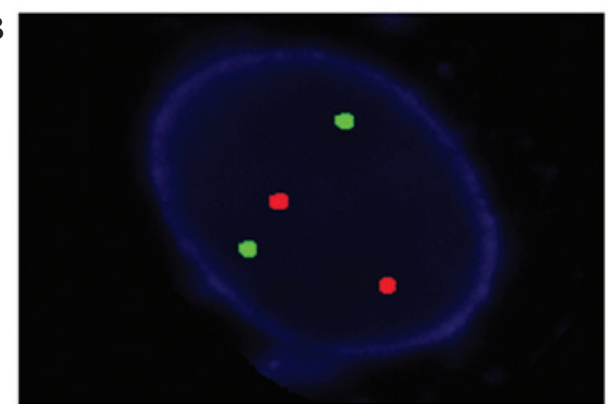

Figure 3. Fluorescence in situ hybridization of urine exfoliated cells following surgery did not reveal any duplications of (A) chromosomes 17 (green) and 9 p16 (red) or (B) chromosomes 3 (green) and 7 (red).

as well as negative reactivity for cytokeratins 7,8 and 18 , confirming the diagnosis of paraganglioma (Fig. 2B, C and D). Following surgery, FISH of exfoliated cells in the urine showed no chromosomal duplications (Fig. 3). Further treatment was avoided as no tumor cells were observed in the deep muscle layer. At 2 years after the surgery, the patient remained 
disease-free. Written informed consent was obtained from the patient for inclusion in the present study.

\section{Discussion}

Histological and immunohistochemical staining is often used for the definitive diagnosis of paragangliomas; however, their determination of malignant potential remains a challenge for urologists and pathologists (6). Chromosomal imbalances have become a new parameter to predict the assessment of malignant potential of paraganglioma (7). As summarized by Schaefer et al (7), gains or losses of chromosomes 1, 3, 6, $7,8,9,11,16,17,19,20,21$ and 22 in paragangliomas have been reported. Furthermore, a gain of $17 \mathrm{p}$ has been implicated in increased probability of progression to malignancy (8). A previous comparative genomic hybridization study was the first to identify that paraganglioma of the urinary bladder shares common chromosomal imbalances with other paragangliomas and related pheochromocytomas (7). Similarly, multiprobe FISH analysis of exfoliated cells in the urine may identify specific abnormalities in chromosomes 3 and 17, in addition to others (9). As a non-invasive method, it has been approved for use in the detection of recurrent urothelial carcinoma and the evaluation of hematuria (9). In the current patient, chromosome duplications in paraganglioma tissue were detected by FISH in exfoliated cells in the urine, which is helpful to determine the malignant potential of paraganglioma. However, the precise association between chromosome duplications and the malignant potential of paraganglioma requires further research.

In the present case, the submucosal neoplasm of the urinary bladder with intense uniform enhancement, and the results of the preoperative histological analysis, were indicative of hemangioma. However, duplications of chromosomes 3 or 17 have not been reported in cases of hemangioma of the urinary bladder, providing a clue for their differential diagnosis. Furthermore, bladder neoplasms with positive FISH results are commonly considered to be urothelial carcinomas (9). It is indicated that submucosal tumors, such as paraganglioma, with chromosome duplications and ulceration, may shed into the urine and be detected. Thus, if FISH of urine exfoliated cells from a patient with a neoplasm of the urinary system, such as a urothelial carcinoma and submucosal tumor, reveals positive results, then chromosome duplication and ulceration should be considered, as shown in the current case report.

In conclusion, the preoperative diagnosis and malignant potential predication of urinary bladder paraganglioma present significant challenges for urologists, and is currently limited to evaluation of specific symptoms, imaging investigations and histological analysis of tissue biopsies. As in the current case, chromosome duplications of urinary bladder paraganglioma detected by FISH in urine exfoliated cells have the potential to provide additional information. This may facilitate the differential diagnosis and malignant determination of patients with urinary bladder paraganglioma.

\section{Acknowledgements}

The authors would like to thank Professor Hua Xu (Department of Urology, Tongji Hospital, Tongji Medical College, Huazhong University of Science and Technology) for technical assistance in the analysis of the FISH studies.

\section{References}

1. Leestma JE and Price EB Jr: Paraganglioma of the urinary bladder. Cancer 28: 1063-1073, 1971.

2. Ansari MS, Goel A, Goel S, Durairajan LN and Seth A: Malignant paraganglioma of the urinary bladder. A case report. Int Urol Nephrol 2001 33: 343-345, 2001.

3. Lai Y, Chen D, Yu Z, Ni L and Yang S: Non-functioning paraganglioma of the urinary bladder: A case report and review of the literature. Oncol Lett 7: 891-893, 2014.

4. Stallings RL: Are chromosomal imbalances important in cancer? Trends Genet 23: 278-283, 2007.

5. Halling KC, King W, Sokolova IA, Meyer RG, Burkhardt HM, Halling AC, Cheville JC, Sebo TJ, Ramakumar S, Stewart CS, et al: A comparison of cytology and fluorescence in situ hybridization for the detection of urothelial carcinoma. J Urol 164: 1768-1775, 2000.

6. Kang WY, Shen JT and Chai CY: Paraganglioma of the urinary bladder: A case report. Kaohsiung J Med Sci 19: 136-140, 2003.

7. Schaefer IM, Gunawan B, Füzesi L, Blech M, Frasunek J and Loertzer H: Chromosomal imbalances in urinary bladder paraganglioma. Cancer Genet Cytogenet 203: 341-344, 2010.

8. August C, August K, Schroeder S, Bahn H, Hinze R, Baba HA, Kersting C and Buerger H: CGH and CD 44/MIB-1 immunohistochemistry are helpful to distinguish metastasized from nonmetastasized sporadic pheochromocytomas. Mod Pathol 17: $1119-1128,2004$.

9. Caraway NP and Katz RL: A review on the current state of urine cytology emphasizing the role of fluorescence in situ hybridization as an adjunct to diagnosis. Cancer Cytopathol 118: 175-183, 2010. 